\title{
The effect of verbal and video feedback on learning direct laryngoscopy among novice laryngoscopists: a randomized pilot study
}

\section{L'effet des rétroactions verbales et par vidéo sur l'apprentissage de la laryngoscopie directe chez les nouveaux laryngoscopistes: une étude pilote randomisée}

\author{
Jennifer E. Sainsbury, MBBS • Branislav Telgarsky, MD 1 Matteo Parotto, MD, PhD • \\ Ahtsham Niazi, MBBS · David T. Wong, MD • Richard M. Cooper, MSc, MD \\ Received: 28 April 2016/Revised: 10 October 2016/Accepted: 8 December 2016/Published online: 27 December 2016 \\ (C) Canadian Anesthesiologists' Society 2016
}

\begin{abstract}
Purpose Skill acquisition in direct laryngoscopy $(D L)$ and tracheal intubation is complex. This pilot study aims to assess feasibility and determine sample size for a subsequent trial comparing DL instruction using a Macintosh-style video laryngoscope (MacVL), with and without video recordings, with conventional $D L$ instruction.

Methods Medical students with no prior laryngoscopy experience were recruited during their two-week anesthesia rotation. During the first (TRAINING) week, students were randomized into three groups: Control (Macintosh direct laryngoscope), VL-1 (MacVL with realtime feedback), and VL-2 (MacVL with real-time feedback plus video recordings of laryngoscopies). During the second (TESTING) week, all students were tested using a Macintosh direct laryngoscope. Feasibility objectives were
\end{abstract}

Jennifer E. Sainsbury and Branislav Telgarsky share the first authorship.

Presented in part at the World Airway Management Meeting (WAMM) 2015, Dublin November 2015 "Does Video Laryngoscopy Accelerate Learning Of Direct Laryngoscopy?"

This pilot study is not registered in a public registry of clinical trials.

J. E. Sainsbury, MBBS - B. Telgarsky, MD - M. Parotto, MD, $\mathrm{PhD} \cdot$ R. M. Cooper, MSc, MD ( $\square)$

Department of Anesthesia, Toronto General Hospital, University Health Network, University of Toronto, Toronto, ON, Canada e-mail: richard.cooper@uhn.ca

A. Niazi, MBBS · D. T. Wong, MD

Department of Anesthesia, Toronto Western Hospital, University

Health Network, University of Toronto, Toronto, ON, Canada recruitment and attrition rates, ability to time and video record intubations, and the availability of a MacVL. The primary clinical outcome during the TESTING week was total time to intubate, and secondary outcomes included intubation success rate, intubating opportunities, complications, and confidence scores.

Results Sixty-eight of 87 (78\%) consecutive medical students approached to participate in the study were recruited over 18 months. Eight (12\%) students withdrew from the study, and data are available on the remaining 60 participants. The times to intubate were recorded for $92 \%$ of the TESTING intubations, but only $71 \%$ of the TRAINING intubations in the VL-2 group were video recorded. The MacVLs were available in 100\% of cases. We estimate that 190 participants would be required for a study restricted to a comparison of DL vs video laryngoscopy with real-time feedback.

Conclusion This pilot study establishes feasibility and provides a sample size estimate for a future RCT. Required modifications to the study protocol include wider hospital involvement and consideration regarding standardization of airway education, teaching, feedback, and patient characteristics.

\section{Résumé}

Objectif L'acquisition de compétences en laryngoscopie directe et en intubation trachéale est complexe. L'objectif de cette étude pilote était d'évaluer la faisabilité et de déterminer la taille d'échantillon nécessaire à réaliser une étude subséquente comparant l'enseignement de la laryngoscopie directe à l'aide d'un vidéolaryngoscope avec une lame de Macintosh (MacVL), avec ou sans 
enregistrement vidéo, à un enseignement traditionnel de la laryngoscopie directe.

Méthode Des étudiants en médecine n'ayant aucune expérience préalable de laryngoscopie ont été recrutés pendant leur rotation d'anesthésie de deux semaines. Pendant la première semaine (FORMATION), les étudiants ont été randomisés en trois groupes: le groupe témoin (laryngoscopie direct avec lame de Macintosh), le groupe VL-1 (MacVL avec rétroaction en temps réel), et $V L-2$ (MacVL avec rétroaction en temps réel et enregistrements vidéo des laryngoscopies). Pendant la deuxième semaine (ÉVALUATION), tous les étudiants ont été évalués en utilisant un laryngoscope direct avec une lame de Macintosh. Les objectifs de faisabilité étaient les taux de recrutement et d'abandon, la capacité à mesurer la durée et à réaliser des enregistrements vidéo des intubations, et la disponibilité d'un MacVL. Le critère d'évaluation principal clinique pendant la semaine d'ÉVALUATION était le temps total jusqu'à intubation, et les critères secondaires étaient le taux de réussite d'intubation, les occasions d'intubation, les complications et les scores de confiance.

Résultats Soixante-huit des 87 (78\%) étudiants en médecine consécutifs auxquels on a demandé de participer à l'étude ont été recrutés au cours d'une période de 18 mois. Huit (12\%) étudiants se sont retirés de l'étude, et des données étaient disponibles pour les 60 participants restants. Les temps jusqu'à intubation ont été enregistrés dans $92 \%$ des intubations réalisées durant l'ÉVALUATION, mais seules $71 \%$ des intubations de la semaine de FORMATION ont été enregistrées dans le groupe VL-2. Les MacVL étaient disponibles dans $100 \%$ des cas. Nous estimons qu'il faudrait 190 participants pour réaliser une étude se limitant à comparer la laryngoscopie directe vs la vidéolaryngoscopie avec rétroaction en temps réel.

Conclusion Cette étude pilote a déterminé la faisabilité et propose un estimé de la taille d'échantillon nécessaire à une future étude randomisée contrôlée. Certaines modifications seraient nécessaires au protocole de l'étude, notamment une implication plus importante dans l'hôpital ainsi que des considérations quant à la standardisation de la formation sur les voies aériennes, l'enseignement, les rétroactions et les caractéristiques du patient.

Direct laryngoscopy (DL) and tracheal intubation are essential but complex clinical skills. New residents typically show a rapid improvement during the first 20 attempts. Nevertheless, a mean of 45-57 attempts is required to reach a $90 \%$ success rate, ${ }^{1-3}$ and some residents may not achieve an $80 \%$ level of competence despite 100 attempts. $^{4}$ Accordingly, non-anesthesia trainees rarely have the opportunity to perform the requisite intubations. At our university, medical students are required to perform "assisted direct laryngoscopy" as part of their two-week anesthesia rotation and thus may have insufficient opportunity to acquire skill in DL. ${ }^{5}$ This situation presents an opportunity to attempt to accelerate skill acquisition.

Several factors contribute to the limited opportunities for acquiring skill in DL, including other training and experiential expectations, e.g., venous cannulation skills, production pressure in the operating room, patient safety, and wider subspecialty exposure. When DL is being performed, the instructor may assume control and sacrifice a teaching opportunity due to uncertainty about the student's progress. Since the instructor cannot see what the student sees when using DL, it is difficult to provide meaningful feedback. Moreover, if feedback is of limited value and presented at a stressful time for the patient, student, and instructor, the educational value is questionable.

Prior to this study, we observed that many students with limited experience performed successful intubations with a video laryngoscope (VL). Some VLs require a different technique from that of DL, e.g., midline insertion and manipulation of a styletted tracheal tube. Since our training program presumes students will acquire skills in DL, we explored whether Macintosh-style VLs (MacVL), using direct viewing, might provide an opportunity to accelerate DL skill acquisition. The GlideScope Direct ${ }^{\circledR}$ (Verathon Medical, Bothell, WA, USA) and $\mathrm{C}-M A C^{\circledR}$ video laryngoscope (Karl Storz Endoskope, Tuttlingen, Germany) resemble the Macintosh laryngoscope and can be viewed directly or indirectly on a monitor. They appear to increase the success rate of students performing DL while the instructor views the progress on the monitor. ${ }^{6,7}$ This enhanced visualization using the monitor allows the instructor to provide real-time feedback, which is more meaningful and may accelerate skill acquisition. Others have shown that such feedback has a positive impact on subsequent tracheal intubation in mannequins ${ }^{8}$ and patients. ${ }^{9}$ In addition, the laryngoscopy can be video recorded for later review at a time and frequency more conducive to learning.

The use of video review in medical education and skills assessment is increasing; ${ }^{10}$ however, specific investigations in the context of teaching DL would be beneficial. From the educational perspective, a video review can be understood as reflection-on-action and enhancement of self-evaluation. ${ }^{11}$ Analysis of video recordings offers a strategy for future practice, completing Gibbs' reflective cycle for enhanced self-directed lifelong learning. ${ }^{12}$ Recordings increase self- 
awareness and the development of critical thinking ${ }^{13}$ alongside self-assessment skills. ${ }^{14-17}$ We adopted a similar approach when we considered the value of an independent self-review of own MacVL video recordings.

There is a lack of studies comparing the impact of teaching DL to novices using MacVL on actual patients vs using traditional teaching methods. Furthermore, we do not know what impact an independent self-review of tracheal intubation attempts with MacVL may have on learning DL. This pilot study was conducted to assess the feasibility of conducting a larger multicentre randomized-controlled trial (RCT). The objectives of the pilot study were to establish participant recruitment and retention rates, to determine the feasibility of data collection and equipment availability, and to ascertain the magnitude of differences in DL skills achieved in different groups. We then intended to use the results of the pilot study to estimate sample size for a more definitive study.

We expect the future RCT to test the hypothesis that teaching with a Macintosh-style video laryngoscope (MacVL) vs conventional DL instruction during a training phase improves DL performance during a testing phase. We aimed to make a further comparison between this result (i.e., MacVL vs DL) and that of a third group randomized to receive MacVL training and to self-review video recordings of their performance. We hypothesized that the trainer's real-time verbal feedback while viewing the monitor would result in trainees acquiring DL skill earlier than with conventional DL-as reflected in shorter times to perform intubations. Furthermore, we assumed that students who were provided with recordings of their laryngoscopies would acquire even greater benefit.

\section{Methods}

\section{Participants}

Research ethics board (REB) approval was granted in November 2013. The REB waived the need for patient consent, as the students would be performing supervised DL and tracheal intubations on these patients as part of the regular learning objectives-if deemed appropriate by the anesthesiologist and irrespective of their participation in the study. Medical students at the University of Toronto have a mandatory two-week rotation in anesthesia during their third year. Fifty to 60 students rotate through the anesthesia departments at the Toronto General Hospital and Toronto Western Hospital during an academic year. Most of these students have no prior experience with DL and represent an ideal study group of novices.

All third-year medical students rotating through our departments during January 2014 to August 2015 were invited to participate by e-mail, and their written consent was required for enrolment. Inclusion criterion was no prior experience performing tracheal intubations in patients or mannequins.

Study design

This pilot study had two parts, each lasting one week (Figure). For the first (TRAINING) week, a computer algorithm was used to randomize the students into one of the following three groups:

1. CONTROL group - clinical training on patients using a conventional Macintosh direct laryngoscope (DL group);

2. VL-1 group - clinical training on patients using a GlideScope Direct ${ }^{\circledR}$ VL or Storz C-MAC ${ }^{\circledR} \# 3$ blade with real-time verbal feedback;

3. VL-2 group - clinical training on patients using a GlideScope Direct VL or Storz C-MAC \#3 blade with real-time verbal feedback plus a video recording of the laryngoscopy for their self-review.

We chose a convenience sample (i.e., 60 students) large enough to provide useful information about feasibility and recruitment and to sustain what would likely be a lengthy study with a high number of participants. ${ }^{18} \mathrm{We}$ anticipated that 18 months would be required, allowing for incomplete recruitment and attrition. We aimed to allocate twenty students to each group. The GlideScope Direct VL and Storz C-MAC \#3 blade were considered equivalent devices based on the authors' untested clinical impression. These devices were used interchangeably based on availability.

Prior to their rotation, all medical students were expected to read an anesthesia manual with a chapter on airway management. On day 2 of the TRAINING week, a high-fidelity simulation day is provided to all students irrespective of their participation in the study. The simulation includes DL and tracheal intubation in a mannequin. Airway education in the operating room (OR) was consigned to the supervising anesthesiologist and was not formalized or structured.

Students attempted to perform DL regardless of the group to which they were assigned. In the control (DL) group, feedback was based on the students' description of what they were seeing and the instructors' view over the shoulders of their students. In the two VL groups, the monitors were visible only to the supervising anesthesiologists, and feedback was based on the view on the monitor. Anonymized video recordings of the intubation attempts were made in the VL-2 group and provided to students on a flash drive for self-review. These students documented the number of times they viewed each recording. 


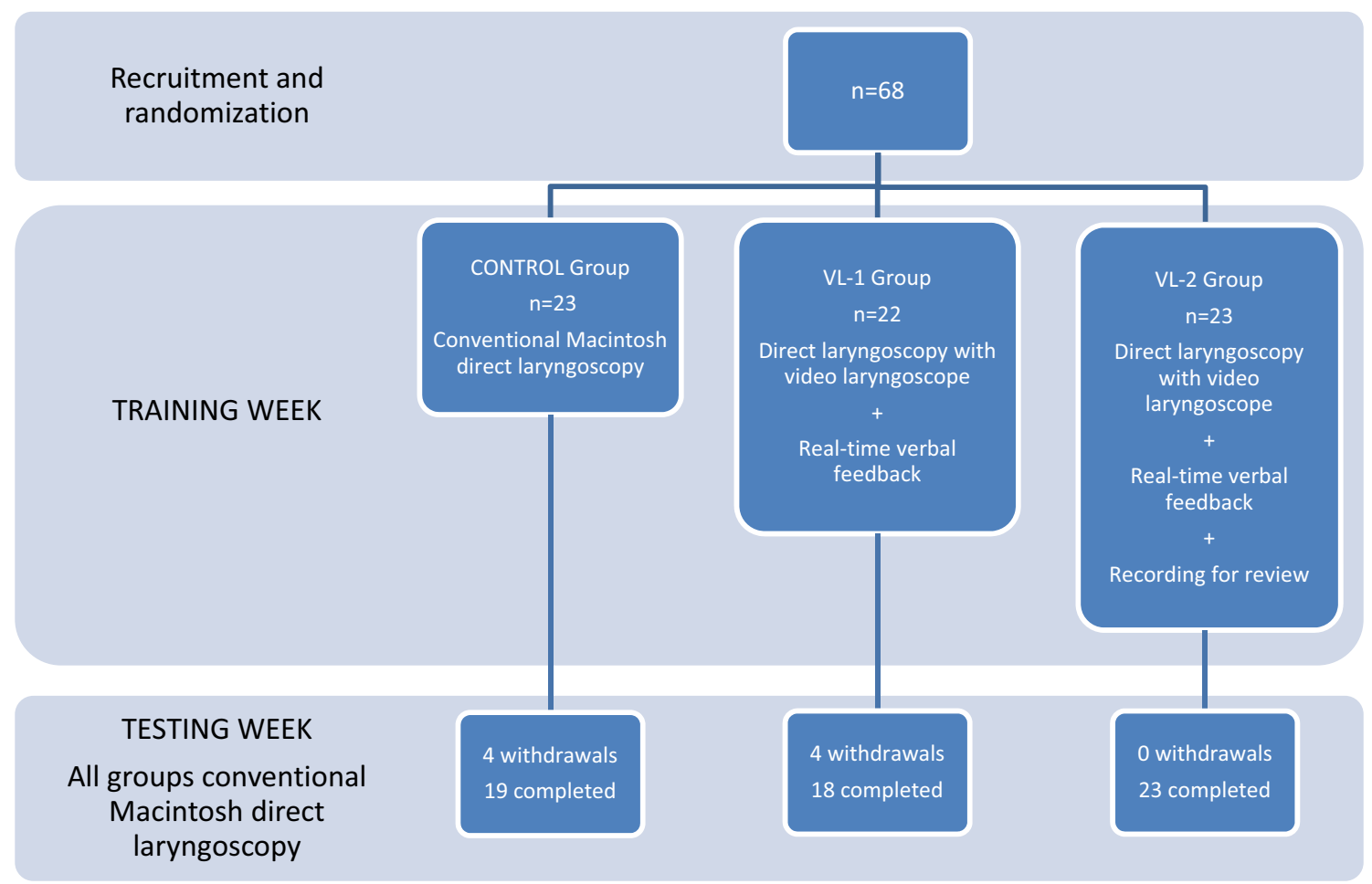

Figure Flowchart of the study

During the second (TESTING) week of the rotation, all three groups were tested using a conventional Macintosh direct laryngoscope. The following data were recorded: patients' sex, age, weight, height, and body mass index; time to intubate (measured from insertion of the laryngoscope into the patient's mouth until a trace of end-tidal carbon dioxide appeared on the monitor); intubation success; and immediate complications. After attempting tracheal intubation, the student and supervising anesthesiologist confidentially documented their subjective confidence in the student's ability to perform DL using a visual analogue scale from 0 (not confident) to 100 (completely confident). Although the anesthesiologist could intervene if patient safety was compromised, two intubating attempts were permitted. The supervising anesthesiologist timed each attempt, whether successful or unsuccessful. To arrive at a single number approximating the efficiency of intubation, time to intubate was considered the total time of up to two intubation attempts. If the student's first attempt was successful, this was regarded as time to intubate. Total times to intubate were then averaged across all participants in the group.

Blinding was not always possible, as the anesthesiologist in the TESTING week may have also supervised the student during the TRAINING week. During both weeks, patients were deemed suitable for student-performed DL based on the clinical discretion of the supervising anesthesiologist without specific inclusion or exclusion criteria related to preoperative airway evaluation. All members of the anesthesia departments were eligible to participate as trainers, were provided with the protocol, and verbally indicated their consent to participate. The anesthesia co-ordinator assigned students to ORs daily based on the presumed educational value. The anesthetic technique was chosen at the discretion of the supervising anesthesiologist without being influenced by participation in the study-patient safety was always prioritized over the needs of the study.

\section{Objectives}

The primary outcome measure was time to intubate during the TESTING week. The secondary outcome measures included 1) intubation success rate during TESTING week, 2) number of intubating opportunities per student per week (individually for each week as well as the number of attempts in TESTING week), 3) complications of DL during the TESTING week, and 4) confidence scores. The statistical analysis was performed using Prism 5.0 (GraphPad Software Inc., La Jolla, CA, USA). Oneway analysis of variance (with Bonferroni's post hoc correction) was used for intubation times, number of intubating opportunities, and difference in students' and supervising anesthesiologists' confidence scores among groups. The Chi square test was used for success rates and 
complication rates. All reported $P$ values are two sided. Correlation between students' and supervising anesthesiologists' confidence scores was assessed by Spearman correlation. Observed differences in means between groups and standard deviations were used to calculate the number of participants needed for the future RCT.

Specific feasibility outcomes for this pilot investigation were not established a priori. Instead, they were determined post hoc following an editorial review recommending their inclusion to comply with standard requirements for a pilot study and to allow a proper assessment of the pilot study findings. The authors considered a recruitment rate $\geq 75 \%$ and an attrition rate of $<20 \%$ to be consistent with feasibility. Based on approximately 60 students rotating through the anesthesia departments annually, this roughly equalled recruiting, on average, four students each month over 18 months and retaining at least three students in the study each month. We had not defined a requisite number of intubations during the training or testing weeks. Other aspects of feasibility were not defined in advance, including our ability to time or record laryngoscopy attempts or the availability of MacVLs during the study.

\section{Results}

Patients' characteristics were similar amongst the groups (Table 1). Sixty-eight (78\%) of the 87 consecutive medical students approached about participation provided written consent and enrolled in the study. Eight (12\%) students withdrew from the study for personal reasons during the TRAINING week without contributing any data. Data are available for the remaining 60 students (Figure). These data satisfy the feasibility outcomes for recruitment rate and attrition rate. Data for time to intubate were recorded in 103/112 (92\%) successful TESTING intubations across all three groups (37/37 Control; 25/29 VL-1; and 41/46 VL-2). Only 78/110 (71\%) TRAINING intubations in the VL-2 group were video recorded, and these 78 recordings were viewed a combined total of 135 times. We did not record a single occurrence of a MacVL being unavailable for a TRAINING intubation in the VL groups (i.e., $100 \%$ equipment availability).

Primary and secondary clinical outcomes are presented in Table 2. Table 3 details analysis of the primary outcome. We found a significant difference in the mean time to intubate between the Control and the two VL Groups (Control, $91 \mathrm{sec}$; VL-1, $61 \mathrm{sec}$; VL-2, $66 \mathrm{sec} ; P=$

Table 1 Demographic data are shown as mean (SD) or as stated

\begin{tabular}{llll}
\hline Patients' characteristics & CONTROL group & VL-1 group & VL-2 group \\
\hline$n$ & 19 & 18 & 23 \\
Age (yr) & $57(13)$ & $58(17)$ & $56(17)$ \\
Female sex & $51 \%$ & $49 \%$ & $49 \%$ \\
BMI & $28(6)$ & $28(6)$ & $28(6)$ \\
\hline
\end{tabular}

Control = Macintosh direct laryngoscope; VL-1 = Macintosh-style video laryngoscope (MacVL) with real-time feedback; VL-2 = MacVL with real-time feedback plus video recordings of laryngoscopies

$\mathrm{BMI}=$ body mass index

Table 2 Results are shown as mean (SD) or as stated

\begin{tabular}{|c|c|c|c|c|}
\hline Outcome variable & $\begin{array}{l}\text { CONTROL group } \\
(n=19)\end{array}$ & $\begin{array}{l}\text { VL-1 group } \\
(n=18)\end{array}$ & $\begin{array}{l}\text { VL-2 group } \\
(n=23)\end{array}$ & $P$ value \\
\hline TRAINING week intubations (per student per week) & $4.7(2.3)$ & $5.3(2.1)$ & $4.8(2.3)$ & 0.69 \\
\hline TRAINING week viewings of each video recording & & & $1.7(1.6)$ & \\
\hline TESTING week intubations (per student per week) & $4.0(1.6)$ & $2.8(1.5)$ & $3.6(1.6)$ & 0.06 \\
\hline $\begin{array}{l}\text { TESTING week total intubations/intubating attempts } \\
\text { (number of attempts per intubation) }\end{array}$ & $77 / 90(1.2)$ & $51 / 59(1.2)$ & $82 / 100(1.2)$ & \\
\hline $\begin{array}{l}\text { TESTING week successful intubations/intubating attempts } \\
\text { (number of attempts per successful intubation) }\end{array}$ & $37 / 43(1.2)$ & $29 / 31(1.1)$ & $46 / 52(1.1)$ & \\
\hline Mean (SD) time to intubate (sec) & $91(62)$ & $61(24)$ & $66(35)$ & $0.018 *$ \\
\hline Intubation success (counts/total intubations) & $48 \%(37 / 77)$ & $57 \%(29 / 51)$ & $56 \%(46 / 82)$ & 0.5 \\
\hline Complications (counts/total intubations) & $25 \%(19 / 77)$ & $19 \%(10 / 51)$ & $15 \%(12 / 82)$ & 0.28 \\
\hline
\end{tabular}

Control = Macintosh direct laryngoscope; VL-1 = Macintosh-style video laryngoscope (MacVL) with real-time feedback; VL-2 = MacVL with real-time feedback plus video recordings of laryngoscopies.*VL-1 vs Control and VL-2 vs Control are statistically significant. SD = standard deviation 
Table 3 Bonferroni post hoc analysis, difference in means and 95\% CI for primary outcome (time to intubate)

\begin{tabular}{llll}
\hline Bonferroni's Multiple Comparison Test & Difference in Means (effect size) $(\mathrm{sec})$ & Significant $(P<0.05) ?$ & $95 \%$ CI \\
\hline CONTROL vs VL-1 & 29.2 & Yes & 0.9 to 57.5 \\
CONTROL vs VL-2 & 24.8 & Yes & 0.1 to 49.6 \\
VL-1 vs VL-2 & -4.4 & No & -32.1 to 23.4 \\
\hline
\end{tabular}

Control = Macintosh direct laryngoscope; VL-1 = Macintosh-style video laryngoscope (MacVL) with real-time feedback; VL-2 = MacVL with real-time feedback plus video recordings of laryngoscopies. CI = confidence interval

0.018). There was no incremental benefit from video reviewing. None of the secondary outcome measures reached statistical significance. Although, with respect to intubation success rates and complication rates, we observed a trend favouring video laryngoscopy over conventional Macintosh direct laryngoscopy as a teaching method. Complications are presented in Table 4. The confidence scores expressed by the students did not correlate with those of the supervising anesthesiologists, and we did not observe any difference among groups.

To calculate the sample size for the future RCT, we considered it clinically relevant to use the difference in means between the two groups of interest (Control vs VL-1). As results from pilot studies may be unreliable for sample size calculations, we exercised great caution in using this observed difference. ${ }^{19}$ Based on the observed difference in means (SD) in time to intubate between Control (DL) and VL-1 in our pilot study, for a probability of type I error $(\alpha)=$ 0.05 and Power $(1-\beta)=0.9$, each group would require 95 students (i.e., total sample size $=190$ ) in a future RCT comparing teaching with DL $v s$ VL with real-time feedback.

\section{Discussion}

This pilot study provided information regarding the value, feasibility, and required sample size for a future RCT. We observed an adequate recruitment $(78 \%)$ and withdrawal $(12 \%)$ rate. The protocol was generally followed with an acceptable level $(92 \%)$ of intubation times recorded. The equipment was consistently available. No significant safety concerns were raised. Technical difficulties or omissions accounted for a $71 \%$ recording rate of TRAINING intubations in the VL-2 group. The low video recording rate, compounded by fewer than two viewings per recording on average (self-reported data by students) and the apparent lack of incremental benefit, will likely result in this cohort being removed, and our subsequent study will be restricted to Control and VL-1 groups. Moreover, as the GlideScope Direct VL is no longer being manufactured, only the Storz C-MAC will be used in the VL group. Recording the confidence scores has also proved unfeasible as these tended to reflect success/failure in individual intubations, precluding any meaningful analysis.

For the sample size calculation for the future RCT, we concluded that each group would require 95 students (i.e., total sample size $=190$ ). These targets are likely achievable in a similar time frame (two years) with the involvement of other teaching institutions. We need to ensure that these hospitals will be compatible with our protocol in terms of student characteristics, recruitment rates, duration of anesthesia rotations, and the availability of equipment. As the RCT will likely be powered for the primary outcome measure only (time to intubate), any potentially statistically significant differences in secondary outcome measures will need to be interpreted with caution (as with most RCTs). If our group or others consider intubation success rate to be a more relevant or preferable outcome, the comparison of the two groups (Control vs VL-1), with a power of $80 \%$, probability of type I error $(\alpha)$ of 0.1 , and a one-sided test, will require 277 students per group. Numbers would increase for three groups (Control vs VL-1 vs VL-2),

Table 4 Complications are shown as numbers

\begin{tabular}{llll}
\hline Complication & $\begin{array}{l}\text { CONTROL } \\
\text { group }\end{array}$ & $\begin{array}{l}\text { VL-1 } \\
\text { group }\end{array}$ & $\begin{array}{l}\text { VL-2 } \\
\text { group }\end{array}$ \\
\hline Placement & 12 & 5 & 8 \\
$\quad$ Endobronchial & 5 & 3 & 1 \\
$\quad$ Esophageal & 7 & 2 & 7 \\
Trauma* & 2 & 1 & 1 \\
Respiratory* & 3 & 1 & 0 \\
Cardiovascular & 2 & 3 & 3 \\
HR $>20 \%$ baseline & 0 & 1 & 1 \\
BP $>20 \%$ baseline & 1 & 2 & 0 \\
HR and BP $>20 \%$ & 1 & 0 & 2 \\
$\quad$ baseline & & & \\
Total & 19 & 10 & 12
\end{tabular}

Control = Macintosh direct laryngoscope; VL-1 = Macintosh-style video laryngoscope (MacVL) with real-time feedback; VL-2 = MacVL with real-time feedback plus video recordings of laryngoscopies. * All trauma complications were damage to lips and all respiratory complications were desaturations $<90 \%$. $\mathrm{BP}=$ blood pressure; $\mathrm{HR}=$ heart rate 
increased power, or decreased type I error. In light of such considerations, the feasibility of such a study may become a relevant issue and will likely require a large multicentre study.

Observed times to intubate in this pilot study are similar to other reported times (i.e., 70-76 sec), ${ }^{9,20}$ though those were single and not combined intubation attempts. There are a number of ways to assess performance of a practical skill. In the case of DL, the most common approaches include overall success rates, ${ }^{8,9}$ first pass success rates, ${ }^{21,22}$ success rates by attempt,${ }^{20}$ intubation times,,${ }^{8,9,20}$ complication rates, ${ }^{9,23}$ and/ or incidence of difficult laryngoscopy. ${ }^{8}$ A combination of these variables likely offers a reasonable assessment of DL performance. We chose time to intubate as our primary outcome as we deemed time required to intubate as best representing the ability to complete an intubation by DL. Real-time feedback enabled our students to achieve success rates of $56 \%$ and $57 \%$ with as few as four or five learning opportunities. Although this is far short of the $90 \%$ used by others to define skill acquisition, ${ }^{2,3}$ there was a trend toward a higher success rate in the VL groups and a clinically meaningful shorter intubation time. In our view, this is an encouraging observation and worthy of further investigation.

\section{Limitations of the study}

Limitations of our study relate to the students, patients, devices, and instructors. As we did not capture students' baseline time to intubate, there may be preexisting differences between groups. We tried to minimize these potential differences by including only those students who had no practical experience with DL as well as by randomization. We were unable to ensure that the patients in the three cohorts were similar with respect to the ease of DL and tracheal intubation. This could easily have introduced a bias that we have not captured. It is unlikely that the lack of universal blinding could have affected the primary outcome, i.e., timing or success of intubation. Nevertheless, it could have had an effect on reporting complications and confidence scores.

The similarity of the direct and indirect views between the GlideScope Direct and the C-MAC may not be identical as we assumed, and the students may not have realized that the view seen on the monitor may have been different from the direct view. ${ }^{24} \mathrm{~A}$ structured approach to self-review or supervised video review could have been useful. In one study, the view on the monitor of the Storz Macintosh video laryngoscope (MVL), an earlier version of C-MAC, was the same as the direct view in $55.8 \%$ of cases, at least one grade better in $41.5 \%$, and worse in $2.7 \% .^{24}$ Thus the supervisor may have been providing real- time verbal feedback that was based on a somewhat different laryngeal view, reducing its value to the student. We acknowledge that the direct and indirect views may not be identical, but we think they afford more meaningful information than the verbal description the student can provide.

Our study design using patients with multiple instructors mirrors the reality of large teaching facilities with feedback from various supervisors with a range of experience. This may have resulted in a greater heterogeneity of outcomes but is likely realistic and generalizable. From an educational perspective, it might have been beneficial to limit the number of instructors and provide formal airway education to the students using multiple modalities (e.g., lectures, videos showing a standard intubation technique, drawings, practice on mannequins, demonstrations) and subsequent practice on patients. ${ }^{20,25}$ In the current climate of scarce resources, other educational approaches may be of interest, e.g., teaching without a teacher ${ }^{26}$ or remote coaching. ${ }^{27}$

\section{Conclusion}

This pilot has established the feasibility, with some modifications, of a subsequent RCT of similar design. We have calculated a sample size and concluded that we would compare only two groups and involve more hospitals. Several considerations are required regarding standardization of airway education, teaching, feedback, and patient characteristics. We trust that the results and lessons learned from this pilot study will serve as a foundation for a future multicentre RCT.

Acknowledgments We sincerely thank Karim Ladha MD and Francesco Cavallin MSc (statistician) for their critical and comprehensive review of the statistical analysis. This study was supported in part by a grant from the Society for Airway Management.

Conflicts of interest No conflicts of interest declared.

Editorial responsibility This submission was handled by Dr. Philip M. Jones, Associate Editor, Canadian Journal of Anesthesia.

Author contributions Jennifer E. Sainsbury, Ahtsham Niazi, David $T$. Wong, and Richard M Cooper were involved in the study design. Jennifer E. Sainsbury, Branislav Telgarsky, Ahtsham Niazi, and David T. Wong were involved in student recruitment. Jennifer E. Sainsbury, Branislav Telgarsky, Matteo Parotto, Ahtsham Niazi, David T. Wong, and Richard $M$ Cooper were involved in data collection. Jennifer E. Sainsbury, Branislav Telgarsky, and Richard M Cooper were involved in writing the article. Branislav Telgarsky, Matteo Parotto, and Richard M Cooper were involved in data analysis. Matteo Parotto, Ahtsham Niazi, and David T. Wong were involved in reviewing the paper. 


\section{References}

1. Kopacz DJ, Neal JM, Pollock JE. The regional anesthesia "learning curve". What is the minimum number of epidural and spinal blocks to reach consistency? Reg Anesth 1996; 21: 182-90.

2. Mulcaster JT, Mills J, Hung OR, et al. Laryngoscopic intubation: learning and performance. Anesthesiology 2003; 98: 23-7.

3. Konrad C, Schupfer G, Wietlisbach M, Gerber H. Learning manual skills in anesthesiology: is there a recommended number of cases for anesthetic procedures? Anesth Analg 1998; 86: 635-9.

4. de Oliveira Filho GR. The construction of learning curves for basic skills in anesthetic procedures: an application for the cumulative sum method. Anesth Analg 2002; 95: 411-6.

5. Niazi A, Matava C. Anesthesia Clerkship Manual - A guide to Anesthesia for Medical Students. Undergraduate Medical Education. University of Toronto, 2016, Toronto UTPress. Available from URL: https://itunes.apple.com/us/book/anesthesiaclerkship-manual/id1151562335? $1 \mathrm{~s}=1 \& \mathrm{mt}=11$ (accessed October 2016).

6. Viernes D, Goldman AJ, Galgon RE, Joffe AM. Evaluation of the GlideScope Direct: a new video laryngoscope for teaching direct laryngoscopy. Anesthesiol Research Pract 2012. DOI:10.1155/ 2012/820961.

7. Kaplan MB, Ward DS, Berci G. A new video laryngoscope - an aid to intubation and teaching. J Clin Anesth 2002; 14: 620-6.

8. Herbstreit F, Fassbender P, Haberl H, Kehren C, Peters J. Learning endotracheal intubation using a novel videolaryngoscope improves intubation skills of medical students. Anesth Analg 2011; 113: 586-90.

9. Howard-Quijano KJ, Huang YM, Matevosian R, Kaplan MB, Steadman $R H$. Video-assisted instruction improves the success rate for tracheal intubation by novices. Br J Anaesth 2008; 101: 568-72.

10. Zick A, Granieri M, Makoul G. First-year medical students' assessment of their own communication skills: a video-based, open-ended approach. Patient Educ Couns 2007; 68: 161-6.

11. Schon DA. Educating the Reflective Practitioner. SF: JosseyBass; 1987 .

12. Gibbs G. Learning by Doing: A Guide to Teaching and Learning Methods. Oxford: Oxford Brookes University; 1998 .

13. Maloney $S$, Paynter $S$, Storr $M$, Morgan $P$. Implementing student self-video of performance. Clin Teach 2013; 10: 323-7.

14. Mort JR, Hansen DJ. First-year pharmacy students' selfassessment of communication skills and the impact of video review. Am J Pharm Educ 2010; 74: 78.
15. Barry S. A video recording and viewing protocol for student group presentations: assisting self-assessment through a Wiki environment. Comput Educ 2012; 59: 855-60.

16. Yoo MS, Yoo IY, Lee H. Nursing students' self-evaluation using a video recording of Foley catheterization: effects on students' competence, communication skills, and learning motivation. J Nurs Educ 2010; 49: 402-5.

17. Jamshidi R, LaMasters T, Eisenberg D, Duh QY, Curet M. Video self-assessment augments development of videoscopic suturing skill. J Am Coll Surg 2009; 209: 622-5.

18. Thabane $L, M a J, C h u R$, et al. A tutorial on pilot studies: the what, why and how. BMC Med Res Methodology 2010; 10: 1.

19. Lenth $R V$. Some practical guidelines for effective sample size determination. Am Stat 2001; 55: 187-93.

20. Ayoub CM, Kanazi GE, Al Alami A, Rameh C, El-Khatib MF. Tracheal intubation following training with the GlideScope compared to direct laryngoscopy. Anaesthesia 2010; 65: 674-8.

21. Sakles JC, Mosier J, Patanwala AE, Dicken J. Learning curves for direct laryngoscopy and GlideScope(R) video laryngoscopy in an emergency medicine residency. West J Emerg Med 2014; 15: 930-7.

22. Brown CA 3rd, Bair AE, Pallin DJ, Walls RM, NEAR Investigators. Techniques, success, and adverse events of emergency department adult intubations. Ann Emerg Med 2015; 65: 363-70.

23. Sakles JC, Javedani PP, Chase E, Garst-Orozco J, GuillenRodriguez JM, Stolz $U$. The use of a video laryngoscope by emergency medicine residents is associated with a reduction in esophageal intubations in the emergency department. Acad Emerg Med 2015; 22: 700-7.

24. Kaplan MB, Hagberg CA, Ward DS, et al. Comparison of direct and video-assisted views of the larynx during routine intubation. $\mathrm{J}$ Clin Anesth 2006; 18: 357-62.

25. Crosby E, Lane A. Innovations in anesthesia education: the development and implementation of a resident rotation for advanced airway management. Can J Anesth 2009; 56: 939-59.

26. Pott LM, Santrock D. Teaching without a teacher: developing competence with a Bullard laryngoscope using only a structured self-learning course and practicing on a mannequin. J Clin Anesth 2007; 19: 583-6.

27. Prescher H, Grover E, Mosier J, et al. Telepresent intubation supervision is as effective as in-person supervision of procedurally naive operators. Telemed J E Health 2015; 21 : $170-5$. 